\title{
Inflammatory Joint Diseases and Risk of Cardiovascular Disease in Modern Rheumatology
}

\section{Inger Jorid Berg 1 (iD) and Sella A. Provan ${ }^{1}$ (iD}

In this issue of The Journal, Liew, et al present a cross-sectional study comparing the 10-year atherosclerotic cardiovascular disease (ASCVD) risk score in patients with axial spondyloarthritis (axSpA) versus the general US population. Their hypothesis was that a diagnosis of axSpA would be associated with a higher risk score of ASCVD ${ }^{1}$.

They studied patients with axSpA participating in 2 different cohort studies (followed at the University of California, San Francisco, and University of Texas Houston Health Science Center). Altogether, the cohorts included patients with both radiographic axSpA/ankylosing spondylitis (AS) and nonradiographic axSpA. Patients were followed prospectively with regular data collections. The 10-year ASCVD risk scores were calculated for patients aged 40-75 years without a history of ASCVD and with available measures of blood pressure and laboratory measures of cholesterol. Individuals from The National Health and Nutrition Examination Survey (NHANES) were used as a comparator group and were matched 4:1 to the axSpA patients according to age, sex, and race.

After calculating the 10-year ASCVD risk scores for both the axSpA group and the NHANES group, the authors subsequently compared the prevalence ratio for a 10-year ASCVD risk score $\geq 7.5 \%$ between the patients with axSpA and the comparator NHANES group, first for the whole axSpA group, and then for the patients with AS (sensitivity analyses).

The authors found that the prevalence ratio of the 10-year ASCVD risk score $\geq 7.5 \%$ was neither increased in patients with axSpA nor in patients with AS compared to the NHANES controls; this finding was in contrast to their hypothesis that

S.A. Provan receives research grant from Southern and Eastern Norway Regional Health Authority.

${ }^{I}$ I.J. Berg, MD, PhD, Consultant Rheumatologist; S.A. Provan, $M D, P h D$

Associate Professor, Consultant Rheumatologist, Department of

Rheumatology, Diakonhjemmet Hospital, Oslo, Norway.

Address correspondence to Dr. I.J. Berg, Diakonhjemmet Hospital,

Department of Rheumatology, Box 23 Vinderen, 0319 Oslo, Norway.

Email:ingerjoridberg@gmail.com.

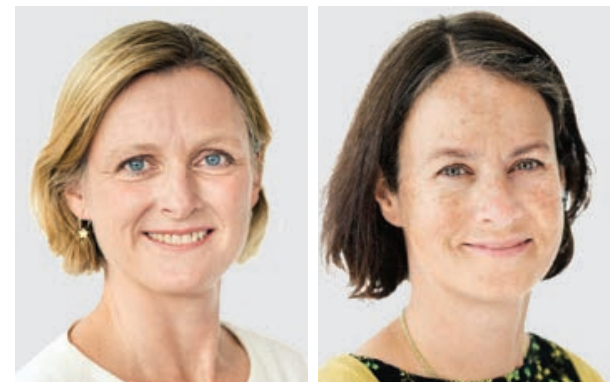

patients with axSpA would have higher ASCVD risk scores. As an explanation of this finding, the authors suggest that the study may be underpowered to find a true difference in ASCVD risk. Alternatively, the results may reflect that the ASCVD risk score underestimates the risk of cardiovascular disease (CVD) in this patient group.

The baseline characteristics showed that there were more smokers and patients with diabetes among the comparators than among the axSpA patients, but analyses restricted to nonsmokers did not alter results. There were no obvious differences in the use of antihypertensives and lipid-lowering medication between the groups. Regarding the use of medication for axSpA, $48 \%$ of the patients used biologics and 65\% used nonsteroidal antiinflammatory drugs (NSAID).

It has long been established that patients with radiographic axSpA have increased CV morbidity and mortality compared to the general population ${ }^{2,3}$. Similarly, patients with other inflammatory joint diseases (IJD), especially rheumatoid arthritis (RA) but also psoriatic arthritis (PsA), have been shown to have increased CVD morbidity and mortality ${ }^{2,4,5}$.

The increased CV morbidity and mortality are most elucidated in RA, and is probably best explained by a complex interplay of several traditional and nontraditional risk factors of $\mathrm{CVD}^{6,7}$. Some traditional risk factors of CVD, such as hypertension (HTN) and obesity, are more frequent among patients with IJD than in the general population, thus enhancing the risk of $\mathrm{CVD}^{2,6}$. Hyperlipidemia, with elevated total cholesterol and low-density lipoprotein cholesterol, also contributes to CVD in patients with IJD, but the lipid levels in patients with IJD are not found to be elevated compared to the general population ${ }^{5,8}$. Interpreting the lipid levels is, however, complex in IJD, where there is an interaction between lipids and inflammation, resulting in lower lipids in patients with high-grade inflammation ${ }^{5}$. Moreover, in active RA, lower lipids have been found to be associated with increased risk of CVD. This phenomenon is referred to as the lipid paradox and is one of the mechanisms by which

See AxSpA CV risk, page 361 
ASCVD risk score calculators may underestimate the risk of CVD in patients with inflammation?

Another important traditional risk factor of CVD is low level of physical activity ${ }^{10}$. Patients with RA and AS have reported less physical activity than population controls ${ }^{6,11}$, even though exercise is recommended as basic treatment of AS. Important barriers for physical activity were pain, stiffness, reduced physical function, and fatigue ${ }^{12}$.

Use of pain killers such as NSAID in the general population has been shown to be associated with increased risk of CVD ${ }^{13}$, and the frequent use of NSAID in patients with IJD might also contribute to increased frequency of $\mathrm{CVD}^{14}$. Moreover, use of steroids, an alternative to NSAID in some cases, may also be associated with increased CVD ${ }^{15}$.

Over the past decades, the pivotal role of inflammation in the development of atherosclerosis has become established ${ }^{7}$. In $\mathrm{RA}$, inflammation and disease activity are found to be associated with $\mathrm{CVD}^{7,16}$. Although the inflammatory burden in patients with axSpA is probably less than in patients with RA, chronic inflammation is believed to enhance the atherosclerotic process, resulting in increased CVD morbidity and mortality ${ }^{2,15}$.

To reduce CV morbidity and mortality in patients with IJD and in the general population, it is important to identify patients with increased risk of CVD. Identifying risk can be performed by evaluating traditional CVD risk factors like HTN, hyperlipidemia, obesity, and additional use of CVD risk scores for the general population ${ }^{10,15}$. There are several calculators of CVD risk score, both nationally developed calculators and calculators developed based on data from several countries. In Europe, the European Heart Score is frequently used, whereas in the United States, the Framingham Risk Score calculator was previously used but has since been replaced by the ASCVD Risk Estimator ${ }^{10,17}$. The latter calculates the 10 -year risk of ASCVD, and for a risk score of $\geq 7.5 \%$ to $20 \%$ (intermediate risk of ASCVD), initiation of treatment with statins should be considered ${ }^{18}$. Additional calculators that take into account nontraditional risk factors such as inflammation in the Reynolds Score, or ethnicity and presence of RA in the QRISK score, have also been developed ${ }^{5,10}$.

Although we have not found studies comparing the validity of CVD risk scores in an axSpA population, established risk calculators have been found to underestimate the risk in populations of patients with RA and PsA, while the QRISK was found to overestimate risk in $\mathrm{RA}^{19,20}$. Due to the underestimation by several CVD risk calculators in IJD, The European League Against Rheumatism (EULAR) recommendations for CVD risk management has suggested multiplying the calculated risk by 1.5 to get a more accurate risk ${ }^{15}$. Efforts have been made to develop RA-specific CVD risk calculators; however, the performance of these calculators have not been superior to the calculators for the general population ${ }^{5}$. Liew, et al suggest in their study that development of a prediction score that better reflects axSpA should be considered ${ }^{1}$. Although we see the value of validating the risk score in each IJD population, we believe that these costly and expansive studies may cause unnecessary delays. We know enough to act now.
EULAR has recommended that patients with IJD should be evaluated every fifth year, and that treatment of risk factors should be initiated when indicated as in the general population $^{15}$. Still, several studies have indicated that there are many patients with IJD who are not evaluated for CVD risk and that there is an undertreatment of the CVD risk factors ${ }^{5,21}$.

Since inflammation is believed to be an important cause of enhanced atherosclerosis ${ }^{7}$, reducing inflammation and disease activity is important when aiming to reduce risk of CVD in these patients ${ }^{15}$. With increased focus on treat-to-target strategies and more availability of biologic treatments, especially in the last 10 years, it is likely that the degree of systemic inflammation of patients with IJD has been reduced. The risk of CVD in IJD may be accordingly reduced through decreased disease activity and inflammation ${ }^{5,22,23}$. Moreover, if inflammation is the main cause of increased CVD risk in IJD, one would expect that the increased risk of CVD may be ameliorated in modern rheumatology, where patients receive more effective inflammation-lowering treatment. In this case, there may not be a true increased frequency of CVD morbidity and mortality in patients with IJD in modern rheumatology $y^{23}$.

As mentioned above, in the results of the study by Liew, et $a l$, the lack of significant differences in the CVD risk scores can indicate that CVD risk in patients with axSpA is underestimated by using CVD risk scores for the general population, given that there is an increased frequency of CVD in patients with axSpA in modern rheumatology ${ }^{1}$. Conversely, finding no significant difference in ASCVD risk score may indicate that with the availability of effective antiinflammatory treatment, there is no increased risk of CVD among patients with axSpA. In the Liew, et als study, $48 \%$ of the axSpA patients were on biologics, which indicates a well-treated patient group using modern strategies ${ }^{1}$.

Still, CVD is a health problem both among patients with IJD and in the general population, and is one of the leading causes of death ${ }^{10}$. Both patients with rheumatic diseases and those in the general population should be evaluated for CVD risk regularly, and treatment should be initiated when indicated. Some earlier studies have, as mentioned above, indicated that there is undertreatment of HTN and hyperlipidemia in patients with IJD. However, in the study by Liew, et al, the blood pressure and cholesterol levels, as well as the proportion taking antihypertensive or lipid-lowering medications, seem to be similar between patients and controls, suggesting that there is no obvious undertreatment of CVD risk factors ${ }^{1}$. This is a possible result of greater focus on CVD in IJD in recent years.

In summary, it has been found previously that increased CV morbidity and mortality are caused by insufficiently targeting patients with IJD, which are diseases related to enhanced atherosclerosis caused by systemic inflammation, and by underestimating or not identifying traditional CVD risk factors through ASCVD risk calculators. To reduce $\mathrm{CV}$ morbidity and mortality in patients with IJD, it is important to follow both of these strategies in the future to reduce CVD: optimal antiinflammatory treatment, and regular evaluations for CVD risk factors as well as initiation of treatment of CVD risk factors when indicated. 


\section{REFERENCES}

1. Liew JW, Reveille JD, Castillo M, Sawhney H, Naovarat BS, Heckbert SR, et al. Cardiovascular risk scores in axial spondyloarthritis versus the general population: a cross-sectional study. J Rheumatol 2021;48:361-6.

2. Papagoras C, Voulgari PV, Drosos AA. Atherosclerosis and cardiovascular disease in the spondyloarthritides, particularly ankylosing spondylitis and psoriatic arthritis. Clin Exp Rheumatol 2013;31:612-20.

3. Haroon NN, Paterson JM, Li P, Inman RD, Haroon N. Patients with ankylosing spondylitis have increased cardiovascular and cerebrovascular mortality: a population-based study. Ann Intern Med 2015;163:409-16.

4. Avina-Zubieta JA, Thomas J, Sadatsafavi M, Lehman AJ, Lacaille D. Risk of incident cardiovascular events in patients with rheumatoid arthritis: a meta-analysis of observational studies. Ann Rheum Dis 2012;71:1524-9.

5. Semb AG, Ikdahl E, Wibetoe G, Crowson C, Rollefstad S. Atherosclerotic cardiovascular disease prevention in rheumatoid arthritis. Nat Rev Rheumatol 2020;16:361-79.

6. Liao KP, Solomon DH. Traditional cardiovascular risk factors, inflammation and cardiovascular risk in rheumatoid arthritis. Rheumatology 2013;52:45-52.

7. Sattar N, McCarey DW, Capell H, McInnes IB. Explaining how "high-grade" systemic inflammation accelerates vascular risk in rheumatoid arthritis. Circulation 2003;108:2957-63.

8. Mathieu S, Gossec L, Dougados M, Soubrier M. Cardiovascular profile in ankylosing spondylitis: a systematic review and meta-analysis. Arthritis Care Res 2011;63:557-63.

9. Myasoedova E, Crowson CS, Kremers HM, Roger VL, Fitz-Gibbon PD, Therneau TM, et al. Lipid paradox in rheumatoid arthritis: the impact of serum lipid measures and systemic inflammation on the risk of cardiovascular disease. Ann Rheum Dis 2011;70:482-7.

10. Piepoli MF, Hoes AW, Agewall S, Albus C, Brotons C, Catapano AL, et al. 2016 European guidelines on cardiovascular disease prevention in clinical practice: the Sixth Joint Task Force of the European Society of Cardiology and other societies on cardiovascular disease prevention in clinical practice (constituted by representatives of 10 societies and by invited experts)developed with the special contribution of the European Association for Cardiovascular Prevention \& Rehabilitation (EACPR). Eur Heart J 2016;37:2315-81.

11. Fongen C, Halvorsen S, Dagfinrud H. High disease activity is related to low levels of physical activity in patients with ankylosing spondylitis. Clin Rheumatol 2013;32:1719-25.

12. Fongen C, Sveaas SH, Dagfinrud H. Barriers and facilitators for being physically active in patients with ankylosing spondylitis: a cross-sectional comparative study. Musculoskeletal Care 2015; 13:76-83.
13. Kearney PM, Baigent C, Godwin J, Halls H, Emberson JR, Patrono C. Do selective cyclo-oxygenase-2 inhibitors and traditional non-steroidal anti-inflammatory drugs increase the risk of atherothrombosis? Meta-analysis of randomised trials. BMJ 2006;332:1302-8.

14. Essers I, Stolwijk C, Boonen A, De Bruin ML, Bazelier MT, de Vries F, et al. Ankylosing spondylitis and risk of ischaemic heart disease: a population-based cohort study. Ann Rheum Dis 2016;75:203-9.

15. Agca R, Heslinga SC, Rollefstad S, Heslinga M, McInnes IB, Peters $\mathrm{MJ}$, et al. Eular recommendations for cardiovascular disease risk management in patients with rheumatoid arthritis and other forms of inflammatory joint disorders: 2015/2016 update. Ann Rheum Dis 2017;76:17-28.

16. Solomon DH, Reed GW, Kremer JM, Curtis JR, Farkouh ME, Harrold LR, et al. Disease activity in rheumatoid arthritis and the risk of cardiovascular events. Arthritis Rheumatol 2015;67:1449-55.

17. Goff DC Jr, Lloyd-Jones DM, Bennett G, Coady S, D’Agostino RB, Gibbons R, et al; American College of Cardiology/American Heart Association Task Force on Practice Guidelines. 2013 ACC/AHA guideline on the assessment of cardiovascular risk: a report of The American College of Cardiology/American Heart Association task force on practice guidelines. Circulation 2014;25 Suppl 2:S49-73.

18. Grundy SM, Stone NJ, Bailey AL, Beam C, Birtcher KK, Blumenthal RS, et al. 2018 AHA/ACC/AACVPR/AAPA/ABC/ ACPM/ADA/AGS/APHA/ASPC/NLA/PCNA guideline on the management of blood cholesterol: a report of the American College of Cardiology/American Heart Association task force on clinical practice guidelines. Circulation 2019;139:e1082-143.

19. Arts EE, Popa C, Den Broeder AA, Semb AG, Toms T, Kitas GD, et al. Performance of four current risk algorithms in predicting cardiovascular events in patients with early rheumatoid arthritis. Ann Rheum Dis 2015;74:668-74.

20. Ernste FC, Sánchez-Menéndez M, Wilton KM, Crowson CS, Matteson EL, Maradit Kremers H. Cardiovascular risk profile at the onset of psoriatic arthritis: A population-based cohort study. Arthritis Care Res 2015;67:1015-21.

21. Molto A, Etcheto A, van der Heijde D, Landewé R, van den Bosch F, Bautista Molano W, et al. Prevalence of comorbidities and evaluation of their screening in spondyloarthritis: results of the international cross-sectional ASAS-COMOSPA study. Ann Rheum Dis 2016;75:1016-23.

22. Ljung L, Askling J, Rantapää-Dahlqvist S, Jacobsson L; ARTIS Study Group. The risk of acute coronary syndrome in rheumatoid arthritis in relation to tumour necrosis factor inhibitors and the risk in the general population: a national cohort study. Arthritis Res Ther 2014;16:R127.

23. Provan SA, Lillegraven S, Sexton J, Angel K, Austad C, Haavardsholm EA, et al. Trends in all-cause and cardiovascular mortality in patients with incident rheumatoid arthritis: A 20-year follow-up matched case-cohort study. Rheumatology 2020; 59:505-12. 